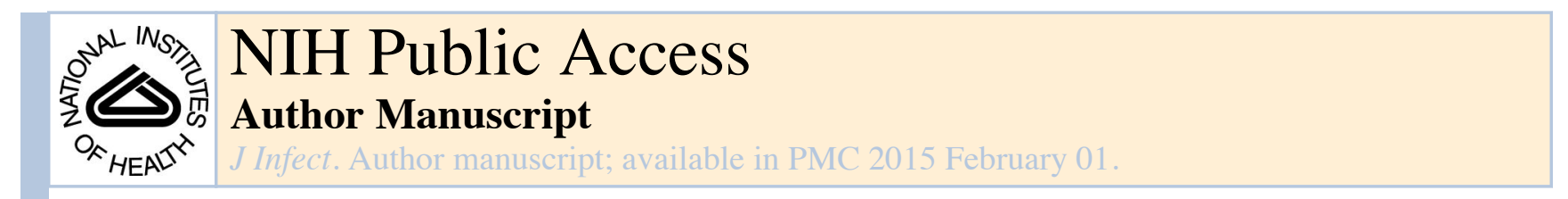

Published in final edited form as:

J Infect. 2014 February ; 68(2): 200-203. doi:10.1016/j.jinf.2013.10.007.

\title{
Injectional anthrax infection due to heroin use induces strong immunological memory
}

\section{Stephanie Ascough,}

Section of Infectious Diseases and Immunity, Department of Medicine, Hammersmith Hospital, Imperial College, London W12 0NN, UK

\section{Rebecca J. Ingram,}

Section of Infectious Diseases and Immunity, Department of Medicine, Hammersmith Hospital, Imperial College, London W12 0NN, UK; Centre for Infection and Immunity, Queen's University Belfast, Belfast, UK

\section{Aula Abarra,}

Section of Infectious Diseases and Immunity, Department of Medicine, Hammersmith Hospital, Imperial College, London W12 ONN, UK

\author{
Alison J. Holmes, \\ Section of Infectious Diseases and Immunity, Department of Medicine, Hammersmith Hospital, \\ Imperial College, London W12 0NN, UK

\section{Bernard Maillere,} \\ Commiseriat à l'Energie Atomique, Insititut de Biologie et de Technologies de Saclay, Service \\ d'Ingénierie Moléculaire des Protéines (SIMOPRO), Gif Sur Yvette, France
}

Daniel M. Altmann, and

Section of Infectious Diseases and Immunity, Department of Medicine, Hammersmith Hospital, Imperial College, London W12 0NN, UK

\section{Rosemary J. Boyton}

Section of Infectious Diseases and Immunity, Department of Medicine, Hammersmith Hospital, Imperial College, London W12 0NN, UK

Dear Sir,

There was a cluster of anthrax cases in heroin users in 2009-2010 and again in 2013, contamination with anthrax of Turkish origin causing often fatal disease. We show here that exposure to anthrax in this manner elicits strong $\mathrm{T}$ cell immunity, even in the face of extremely severe disease. The pattern of immunity seen is reminiscent of immune responses in hyperimmunized vaccines or naturally exposed farmers. This robust response to anthrax is contrary to expectation when one considers the well-documented ablative effect of anthrax toxins on immune function.

\footnotetext{
(C) 2013 The British Infection Association. Published by Elsevier Ltd. All rights reserved.

*Corresponding author. Section of Infectious Diseases and Immunity, Department of Medicine, Hammersmith Hospital, Imperial College, Du Cane Road, London W12 0NN, UK. r.boyton@imperial.ac.uk (R.J. Boyton).
} 
There has been growing concern about infection by spore-forming bacteria leading to softtissue infections and other severe manifestations in heroin users. Clusters of infection derived from contaminated drug batches were seen during 2009-2010 when there were over 80 anthrax cases in Scotland and SE England, seemingly related to a single batch of anthraxcontaminated heroin. ${ }^{1-3}$ There have been a further 13 cases in the UK, Germany, Denmark and France during 2012-2013. ${ }^{4}$ Contamination most likely occurs through exporting drug batches concealed in animal hides, through the manufacturing laboratory containing equipment contaminated with anthrax spores or through heroin having been 'cut' with contaminated material such as powdered bone. Genotypic analysis of the UK cases confirms that all are likely to derive from a single anthrax strain, closely related to strains from Turkey and compatible with the notion of contamination during export to Europe. 5,6

The majority of cases have been fatal. Previous experience of anthrax has been, most commonly, through inhalational contact in 'wool-sorters' disease, ${ }^{7}$ cutaneous exposure in tanners and goat-herders, as well as ingestional exposure through eating infecting livestock. More rarely, there have been instances of deliberate inhalational anthrax exposure, most notoriously the cases following the attacks through the US postal system. ${ }^{8}$ There are no previous reports, outside of animal model experiments, of exposure to anthrax spores through direct injection or by subcutaneous injection ('skin-popping'). These modes of infection are associated with particularly severe and diverse pathological outcomes.

Understanding the bacterial pathogenesis and immunology of anthrax infection is important if we are to develop better strategies for supporting the septic patient exposed to injectional anthrax. Experimental models suggest that the anthrax toxins that target immune cells ablate adaptive immunity to the bacterium. ${ }^{9}$ We show here however, in detailed analysis of $\mathrm{T}$ cell immunity in a survivor of injectional anthrax, that strong immunity can be developed, reminiscent of that seen in vaccinated individuals. ${ }^{10}$

We describe here a 60-year old man who presented to a West London hospital with a fever and left femoral artery pseudoaneurysm one day after injecting heroin into the left groin. There was a pulsatile mass in the left groin; at surgery for the pseudoaneurysm repair, necrotic tissue was debrided. On admission, broad spectrum intravenous antibiotics were started and continued for ten days. These included Ciprofloxacin $500 \mathrm{mg}$ PO BD, Clindamycin 600 mg IV QDS, Flucloxacillin 2 g IV QDS, Benzylpenicillin 2.4 g IV 4 hourly and Metronidazole $500 \mathrm{mg}$ IV TDS. At day 19, further debridement was required and he was recommenced on broad spectrum antibiotics for a further 14 days. Tissue samples taken at this debridement were negative for anthrax on culture and PCR. Serology was subsequently received which was strongly positive. Blood samples were obtained at 5 months after discharge with full, informed consent; ethical approval was obtained under LREC 11/H0721/15. Peripheral blood mononuclear cells (PBMC) were separated by Ficoll Paque centrifugation and CD4 T interferon (IFNgamma) ELIspot responses assessed by culture with overlapping synthetic 20-mer peptides representing the amino acid sequence of anthrax protective antigen (PA) and lethal factor (LF), ${ }^{10}$ final concentration of $25 \mu \mathrm{g} \mathrm{ml}$.

We have previously conducted an extensive analysis of the immunogenicity of epitopes from anthrax PA and LF toxins in agricultural workers from the Kayseri region of Turkey 
who had been hospitalized following occupational exposure to cutaneous anthrax. ${ }^{10-12}$ In that study we found that, despite the extensive literature on the immunotoxic impacts of anthrax, survivors had strong adaptive immune memory, mapping to a number of immunodominant CD4 epitopes. This is likely the reason that reinfection is never seen in those communities. Furthermore, while most efforts regarding protective vaccines have targeted PA, one of the other toxins, LF, was at least as immunogenic in the context of natural infection. Considering the differences between cutaneous anthrax and the very severe clinical picture produced by injectional anthrax, we questioned whether $\mathrm{T}$ cell memory had been established in this individual. We found strong CD4 T cell IFN $\gamma$ responses to epitopes within 4 of the 20 -mer peptides in our PA peptide library and 4 of the peptides in our LF library (Table 1). No responses to these peptides were found in unexposed, healthy control donors. ${ }^{10}$ We related this immune response to other datasets in our lab: PA and LF T cell responses of naturally exposed agricultural workers in Turkey, PA and LF T cell responses of defense workers hyperimmunized with the anthrax vaccine precipitated (AVP) vaccine. We also considered the binding affinity of the anthrax T cell epitopes to common HLA class II alleles in the population, a means of identifying those responses that are of the broadest applicability.

In this individual 4 epitopes from PA elicited strong CD4 T cell responses, PA 41-60, 181$200,451-470$ and 661-680. All of these except 181-200 have previously been seen by us as strong immune epitopes in mapping $\mathrm{T}$ cell immunity in naturally exposed Turkish farmers. We found that PA 41-60 was also strongly immunogenic in HLA class II transgenic mouse strains carrying each of 2 common alleles, HLA-DR1 and HLA-DQ8 (data not shown). PA 181-200 was immunogenic in HLA-DR15 transgenics and PA 661-680 in HLA-DR4 transgenics. In HLA class II peptide binding studies, this latter peptide had the property of binding most alleles tested.

The four epitopes recognized within the LF sequence were LF 41-60, 71-90, 287-306 and 507-526. LF 41-60, 71-90 and 287-306 have previously been noted by us as highly immunogenic in the Turkish cohort. LF 41-60 and 287-306 are also recognized by T cells in AVP vaccinees.

Analysis of adaptive immunity to Bacillus anthracis is of particular interest from the perspective of pathogenesis. However, one might predict that this might be confounded by the mode of action of anthrax toxins: a substantial literature addresses the effects of anthrax toxins on ablation of immune signaling and activation at several different levels - antigen presenting cell function, B cell and T cell signaling. ${ }^{9,12}$ Nevertheless, the patient described here developed strong, potentially protective T cell immunity to several commonly immunodominant epitopes of PA and LF. This response is similar to that of hyper-immunized vaccines or agricultural workers who have recovered from cutaneous anthrax. In experimental models, immunization route can have profound impact on qualitative and quantitative aspects of $\mathrm{T}$ cell immunity, including patterns of epitope recognition. For anthrax exposure, injectional exposure seems to give similar results to intramuscular vaccination or cutaneous contact with animal hides. 
An improved understanding of adaptive immunity to anthrax in these unusual patients has value in consideration of which may be the most immunogenic components of the bacterium for targeting in next-generation vaccines.

\section{Acknowledgments}

This work was supported by funding from National Institutes of Health, National Institute of Allergy and Infectious Diseases, Contract HHSN266200400084C. We are grateful for support from the National Institute for Health Research Biomedical Research funding scheme. The authors wish to acknowledge the NIHR Imperial BRC multiparameter flow cytometry and confocal imaging facility, Hammersmith Campus and the Welton Foundation.

\section{References}

1. Palmateer NE, Ramsay CN, Browning L, Goldberg DJ, Hutchinson SJ. Anthrax infection among heroin users in Scotland during 2009-2010: a case-control study by linkage to a national drug treatment database. Clin Infect Dis. 2012; 55:706-10. [PubMed: 22618565]

2. Hicks CW, Sweeney DA, Cui X, Li Y, Eichacker PQ. An overview of anthrax infection including the recently identified form of disease in injection drug users. Intensive Care Med. 2012; 38:1092104. [PubMed: 22527064]

3. Palmateer NE, Hope VD, Roy K, Marongiu A, White JM, Grant KA, et al. Infections with sporeforming bacteria in persons who inject drugs, 2000-2009. Emerg Infect Dis. 2013; 19:29-34. [PubMed: 23260795]

4. http://www.hpa.org.uk/NewsCentre/NationalPressReleases/2013PressReleases/ 130308SuffolkpatientwithAnthrax/.

5. Price EP, Seymour ML, Sarovich DS, Latham J, Wolken SR, Mason J, et al. Molecular epidemiologic investigation of an anthrax outbreak among heroin users, Europe. Emerg Infect Dis. 2012; 18:1307-13. [PubMed: 22840345]

6. Grunow R, Klee SR, Beyer W, George M, Grunow D, Barduhn A, et al. Anthrax among heroin users in Europe possibly caused by same Bacillus anthracis strain since 2000. Euro Surveill. 2013 Mar 28.18(13)

7. Wattiau P, Govaerts M, Frangoulidis D, Fretin D, Kissling E, Van Hessche M, et al. Immunologic response of unvaccinated workers exposed to anthrax. Belgium Emerg Infect Dis. 2009; 15(10): 1637-40.

8. Dewan PK, Fry AM, Laserson K, Tierney BC, Quinn CP, Hayslett JA, et al. Inhalational anthrax outbreak among postal workers, Washington, D.C., 2001. Emerg Infect Dis. 2002; 8:1066-72. [PubMed: 12396917]

9. Agrawal A, Lingappa J, Leppla SH, Agrawal S, Jabbar A, Quinn C, et al. Impairment of dendritic cells and adaptive immunity by anthrax lethal toxin. Nature. 2003; 424:329-34. [PubMed: 12867985]

10. Ingram RJ, Metan G, Maillere B, Doganay M, Ozkul Y, Kim LU, et al. Natural exposure to cutaneous anthrax gives long-lasting $\mathrm{T}$ cell immunity encompassing infection-specific epitopes. $\mathrm{J}$ Immunol. 2010; 184:3814-21. [PubMed: 20208010]

11. Ingram RJ, Harris A, Ascough S, Metan G, Doganay M, Ballie L, et al. Exposure to anthrax toxin alters human leucocyte expression of anthrax toxin receptor 1. Clin Exp Immunol. 2013; 173:8491. [PubMed: 23607659]

12. Ascough S, Ingram RJ, Altmann DM. Anthrax lethal toxin and the induction of CD4 T cell immunity. Toxins. 2012; 4:878-99. [PubMed: 23162703] 


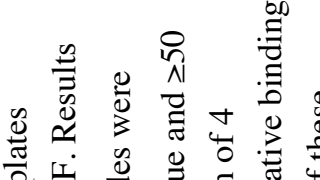

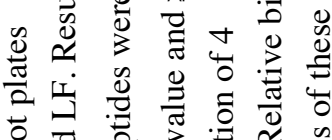

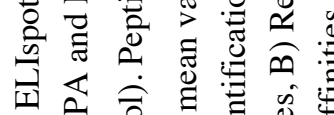

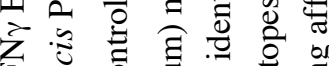

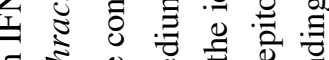

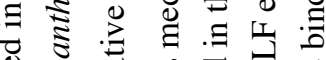

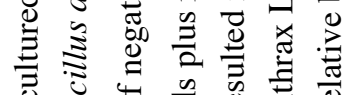

ठ

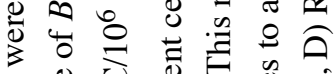

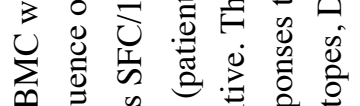

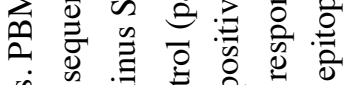

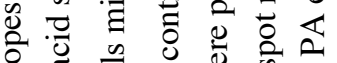

:

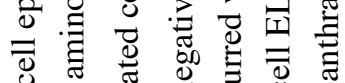

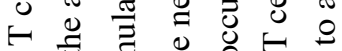

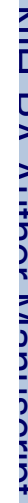

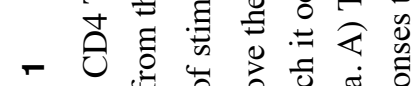

。

-

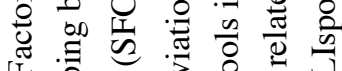
霖

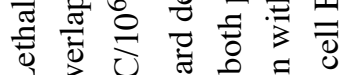

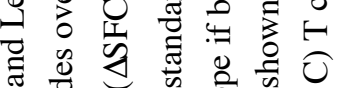

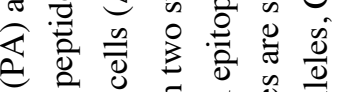

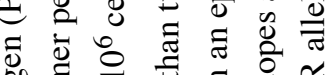

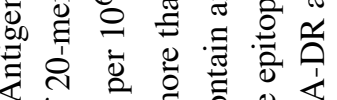

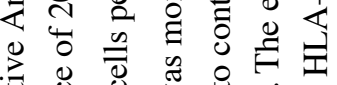

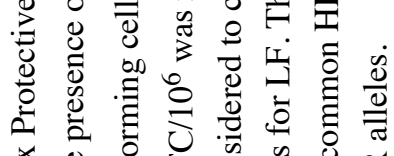

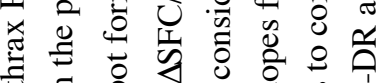

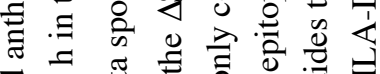

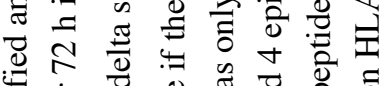

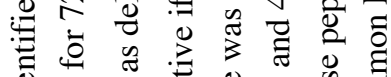

离离离

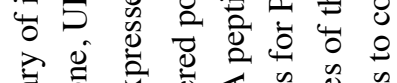

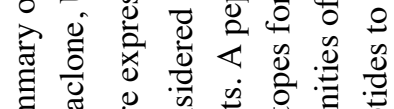

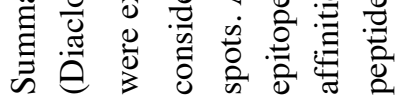

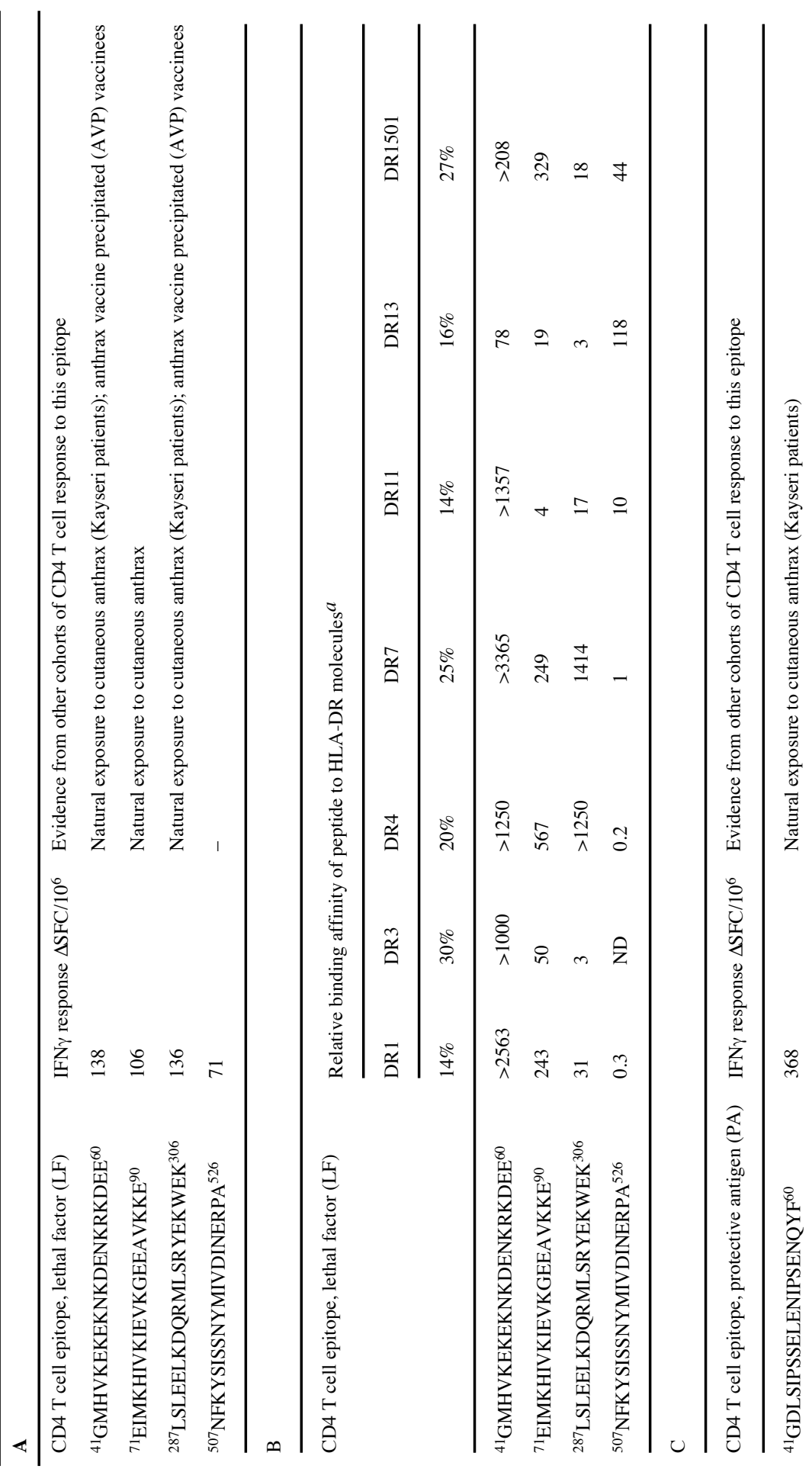




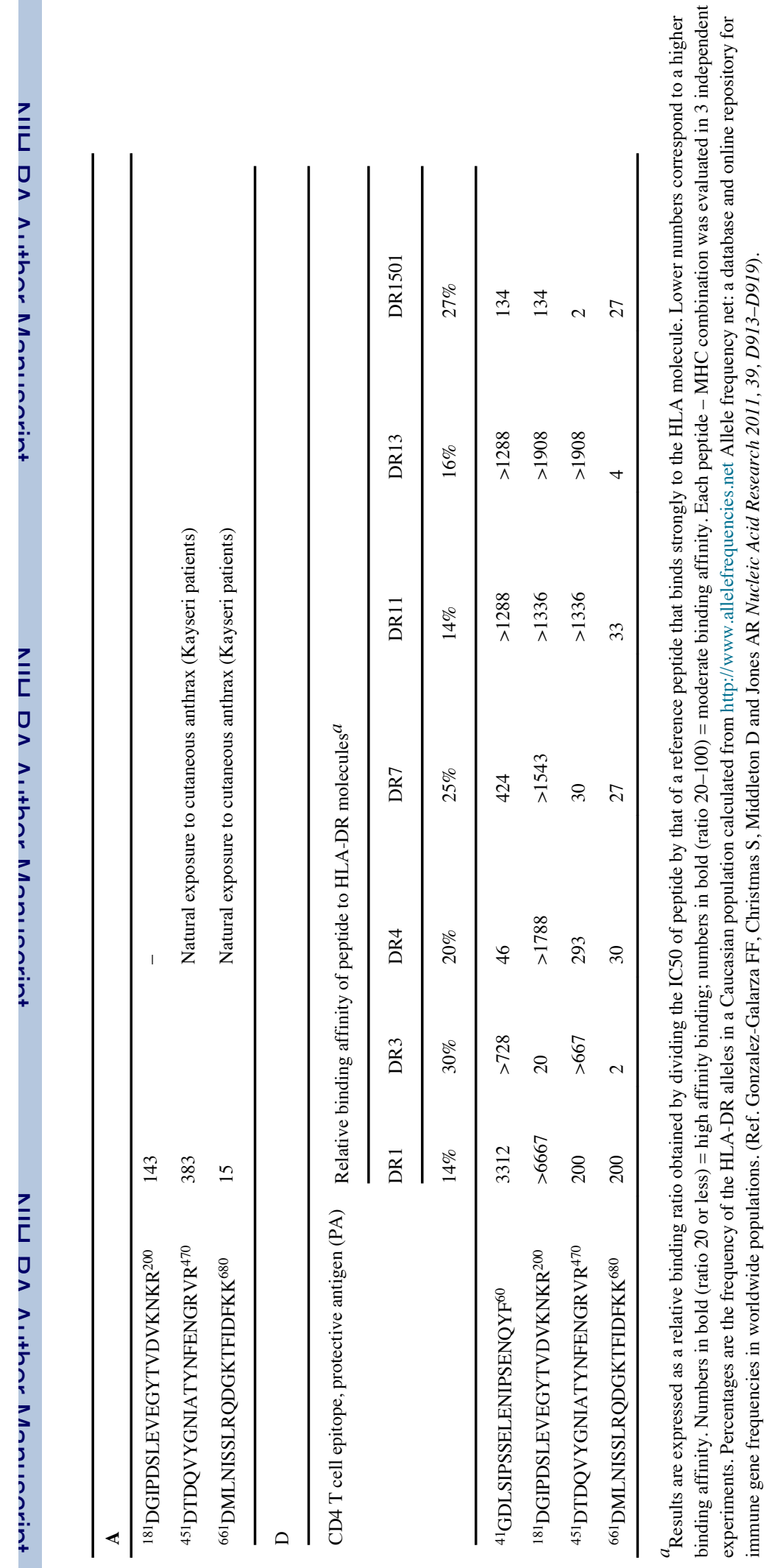

